\title{
On the Golden Rule of capital accumulation under endogenous longevity
}

D. de la Croix and Gr. Ponthiere

Discussion Paper 2008-32

\section{Département des Sciences Économiques de l'Université catholique de Louvain}




\title{
On the Golden Rule of Capital Accumulation under Endogenous Longevity
}

\author{
David de la Croix* Gregory Ponthiere ${ }^{\dagger}$
}

September 25, 2008

\begin{abstract}
This note derives the Golden Rule of capital accumulation in a Chakrabortytype economy, i.e. a two-period OLG economy where longevity is endogenous. It is shown that the capital per worker maximizing steady-state consumption per head is inferior to the Golden Rule capital level prevailing under exogenous longevity. We characterize also the Lifetime Golden Rule, that is, the capital per worker maximizing steady-state expected lifetime consumption per head, and show that this tends to exceed the standard Golden Rule capital level.
\end{abstract}

JEL codes: E13, E21, E22, I12.

Keywords: Golden Rule, longevity, OLG models.

${ }^{*}$ Department of Economics and CORE, Université catholique de Louvain. E-mail: david.delacroix@uclouvain.be. De la Croix acknowledges the financial support of the Belgian French speaking community (Grant ARC 03/08-235 "New Macroeconomic Approaches to the Development Problem") and the Belgian Federal Government (Grant PAI P6/07 "Economic Policy and Finance in the Global Economy: Equilibrium Analysis and Social Evaluation").

${ }^{\dagger}$ PSE and Ecole Normale Suprieure (Paris). Address: ENS, 48 Boulevard Jourdan, 75014 Paris, France. E-mail: gregory.ponthiere@ens.fr 


\section{Introduction}

Introduced by Phelps (1961), the Golden Rule of capital accumulation states the condition under which the stock of capital per worker maximizes steady-state consumption per head. As it is well-known, the Golden Rule states, in a simple model with no technological progress, that steady-state consumption per head is maximized when the marginal productivity of capital equals the sum of the population growth rate and the rate of depreciation of capital.

Whereas the Golden Rule has given rise to various studies in growth theory (see Diamond, 1965; Phelps, 1965), no attention has been paid so far to the Golden Rule of capital accumulation in an economy where agents's lifetime is endogenous, as in Chakraborty's (2004) OLG model. Under such an endogenous (finite) time horizon, does the Golden Rule capital level remain the same, or, on the contrary, does the endogeneity of lifetime modify the Golden Rule?

Given the growing body of recent literature with endogenous longevity, ${ }^{1}$ it is important to determine the Golden Rule capital level in this context, as a benchmark. Accordingly, we use a two-period OLG model with physical capital based on Chakraborty (2004), where the probability of survival to the second period of life depends positively on some public health expenditures. We then explore the definition of the Golden Rule capital level in that context.

The rest of this note is organized as follows. Section 2 presents the model. Section 3 derives the Golden Rule of capital accumulation in an economy with endogenous longevity. Section 4 explores an alternative definition of the Golden Rule, named the Lifetime Golden Rule. Section 5 concludes.

\section{The model}

Let us consider an OLG model with the same population structure and technology as in the model studied by Chakraborty (2004). Time is discrete and goes from 0 to infinity, but households live at best for two periods.

Demography The size of the cohort born at $t$ is $L_{t}$. It grows over time at a constant, exogenous rate $n(n>-1)$ :

$$
L_{t+1}=(1+n) L_{t}
$$

All agents of a cohort $t$ live the first period of life for sure, but only a proportion $\pi_{t+1}$ of that cohort will enjoy a second period of life (which is also of unitary length). ${ }^{2}$ The

\footnotetext{
${ }^{1}$ Cervellati and Sunde (2005) and Chakraborty and Das (2006) are two highly cited examples. A survey is in Boucekkine (2008).

${ }^{2}$ Hence, life expectancy at birth is here equal to $\pi_{t+1} 2+\left(1-\pi_{t+1}\right) 1=1+\pi_{t+1}$.
} 
proportion of survivors $\pi_{t+1}\left(0<\pi_{t+1}<1\right)$ depends positively on the amount of health expenditures per worker $h_{t}$ :

$$
\pi_{t+1}=\pi\left(h_{t}\right)
$$

with $\pi^{\prime}(h)>0$ and $\pi^{\prime \prime}(h)<0$. We also assume that and $\lim _{h_{t} \rightarrow \infty} \pi\left(h_{t}\right)=1$, which gives an upper bound to the life expectancy $1+\pi$.

Technology Firms at time $t$ produce some output $Y_{t}$ according to the following production function: $Y_{t}=F\left(K_{t}, L_{t}\right)$ where $Y_{t}$ denotes the total output, $K_{t}$ the total capital stock, and $L_{t}$ denotes the labour force. $F($.$) is a positively-valued production function,$ increasing, and strictly concave with respect to capital. Capital depreciates at a constant rate $\delta(0 \leq \delta \leq 1)$. Under constant returns to scale, production can be rewritten as:

$$
y_{t}=f\left(k_{t}\right)
$$

where $y_{t}$ denotes the output per worker, and $k_{t}$ the capital stock per worker, while $f()=$. $F(k, 1)$ is the production function in its intensive form. Under the above assumptions on $F($.$) , we have, for all k>0, f(k)>0, f^{\prime}(k)>0$ and $f^{\prime \prime}(k)<0$. (de la Croix and Michel, 2002)

The marginal productivity of capital is equal to $f^{\prime}(k)$. The marginal productivity of labour is given by the function

$$
\omega(k)=f(k)-k f^{\prime}(k)
$$

It can be shown that the marginal productivity of labor $\omega(k)$ satisfies $\omega(k) \geq 0$ and $\omega^{\prime}(k)=-k f^{\prime \prime}(k)>0$.

Government The government runs a balanced budget. It taxes the marginal productivity of labour at a rate $\tau$, and funds public health expenditures $H_{t}: \tau L_{t} \omega\left(k_{t}\right)=H_{t}$ or, in intensive terms:

$$
h_{t}=\tau \omega\left(k_{t}\right)
$$

Note that $h_{t}$ consists merely of a longevity-enhancing expenditure, but does not affect the quality of each period of life.

\section{The Golden Rule}

Consider a stationary environment in which the variables $k, h$ and $\pi$ are constant over time and all the aggregate variables, production $Y_{t}$, consumption $C_{t}$, investment $I_{t}$, health spending $H_{t}$, and capital $K_{t}$ grow at the constant rate $n$. Let us derive the level of capital per worker $k$ maximizing steady-state consumption per head. The feasibility constraint imposes that investment $I_{t}$ is equal to production $F\left(K_{t}, L_{t}\right)$ minus consumption $C_{t}$ minus health spending $H_{t}: I_{t}=K_{t+1}-(1-\delta) K_{t}=F\left(K_{t}, L_{t}\right)-C_{t}-H_{t}$ so 
that total consumption $C_{t}$ is equal to: $C_{t}=F\left(K_{t}, L_{t}\right)-K_{t+1}-H_{t}+(1-\delta) K_{t}$. Thus, consumption per worker, equal to $C_{t} / L_{t}=c_{t}=c$, can be written as:

$$
c=f(k)-k(\delta+n)-\tau \omega(k)
$$

Consumption per head $C_{t} /\left(L_{t}+\pi_{t} L_{t-1}\right)$ where $\pi_{t}=\pi(\tau \omega(k))=\pi$ is related to consumption per worker through the following identity:

$$
\frac{C_{t}}{L_{t}+\pi L_{t-1}}=\frac{c_{t}}{\left(1+\frac{\pi}{1+n}\right)}=c_{t} \frac{1+n}{1+n+\pi}
$$

given that $L_{t}=(1+n) L_{t-1}$. Consumption per head corresponds to consumption per worker $c_{t}$, multiplied by $(1+n) /(1+n+\pi)$. Note that this latter factor depends on $\pi$, and, thus, on capital per worker. Hence, contrary to what prevails in standard OLG models with exogenous longevity, the capital level maximizing consumption per head does not here coincide with the one maximizing consumption per worker.

It follows that consumption per head at the steady-state, denoted by $\phi(k)$, can be written as:

$$
\phi(k)=[f(k)-k(\delta+n)-\tau \omega(k)] \frac{1+n}{1+n+\pi(\tau \omega(k))}
$$

In order to discuss the conditions necessary and sufficient for the existence of a Golden Rule capital level, let us first differentiate consumption per head $\phi(k)$ with respect to capital:

$$
\phi^{\prime}(k)=\left[f^{\prime}(k)-(\delta+n)-\tau \omega^{\prime}(k)\right]-\frac{[f(k)-k(\delta+n)-\tau \omega(k)] \pi^{\prime}(\tau \omega(k)) \tau \omega^{\prime}(k)}{1+n+\pi(\tau \omega(k))}
$$

As this was argued by de la Croix and Michel (2002), the expression $\phi^{\prime}(k)=0$ defines an interior Golden Rule capital level only if $\phi(k)$ is neither always decreasing in $k$ (implying that the capital level maximizing $\phi(k)$ is 0 ), nor always increasing in $k$ (implying that the capital maximizing $\phi(k)$ is infinite). The interiority of the solution requires the following condition, which guarantees that $\phi^{\prime}(k)$ is positive when $k$ tends to 0 , but negative when it tends to $+\infty$.

Proposition 1 Assume that $\{n, \delta, \tau, f(k), \pi(h)\}$ satisfy:

$$
\begin{aligned}
\lim _{k \rightarrow 0+} f^{\prime}(k) & >\delta+n+\lim _{k \rightarrow 0+}\left(\frac{\pi^{\prime}(\tau \omega(k))\left[(1-\tau) f(k)+\tau k f^{\prime}(k)\right]}{1+n+\pi(\tau \omega(k))}+1\right) \tau k\left|f^{\prime \prime}(k)\right| \\
\lim _{k \rightarrow+\infty} f^{\prime}(k) & <\delta+n
\end{aligned}
$$

Then, there exists a capital per worker $k_{\mathrm{GR}}$ maximizing consumption per head in $\mathbb{R}_{+}$. Such a level satisfies $\phi^{\prime}\left(k_{\mathrm{GR}}\right)=0$ :

$$
\frac{f^{\prime}\left(k_{\mathrm{GR}}\right)-(\delta+n)+\tau k_{\mathrm{GR}} f^{\prime \prime}\left(k_{\mathrm{GR}}\right)}{f\left(k_{\mathrm{GR}}\right)-k_{\mathrm{GR}}(\delta+n)-\tau \omega\left(k_{\mathrm{GR}}\right)}=\frac{\tau \omega^{\prime}\left(k_{\mathrm{GR}}\right) \pi^{\prime}\left(\tau \omega\left(k_{\mathrm{GR}}\right)\right)}{1+n+\pi\left(\tau \omega\left(k_{\mathrm{GR}}\right)\right)}
$$


Proof. The conditions $\lim _{k \rightarrow 0+} \phi^{\prime}(k)>0$ and $\lim _{k \rightarrow+\infty} \phi^{\prime}(k)<0$ are sufficient to obtain an interior maximum. The first limit can be written as:

$\lim _{k \rightarrow 0+} \phi^{\prime}(k)=\lim _{k \rightarrow 0+}\left[f^{\prime}(k)-(\delta+n)+\tau k f^{\prime \prime}(k)-\frac{\pi^{\prime}(\tau \omega(k)) \tau \omega^{\prime}(k)\left[(1-\tau) f(k)+\tau k f^{\prime}(k)\right]}{1+n+\pi(\tau \omega(k))}\right]$

That condition $\lim _{k \rightarrow 0+} \phi^{\prime}(k)>0$ can be rewritten as: (using $\left.\omega^{\prime}(k)=-k f^{\prime \prime}(k)\right)$ :

$$
\begin{gathered}
\lim _{k \rightarrow 0+}\left[f^{\prime}(k)-(\delta+n)+\tau k f^{\prime \prime}(k)\right]>\lim _{k \rightarrow 0+}\left[\frac{\pi^{\prime}(\tau \omega(k)) \tau \omega^{\prime}(k)\left[(1-\tau) f(k)+\tau k f^{\prime}(k)\right]}{1+n+\pi(\tau \omega(k))}\right] \\
\lim _{k \rightarrow 0+}\left[f^{\prime}(k)-(\delta+n)\right]>\lim _{k \rightarrow 0+} \tau k\left|f^{\prime \prime}(k)\right|\left(1+\frac{\pi^{\prime}(\tau \omega(k))\left[(1-\tau) f(k)+\tau k f^{\prime}(k)\right]}{1+n+\pi(\tau \omega(k))}\right)
\end{gathered}
$$

which is the condition in the Proposition. Regarding the second condition, we have:

$$
\begin{aligned}
& \lim _{k \rightarrow \infty} \phi^{\prime}(k)=\lim _{k \rightarrow \infty}\left[f^{\prime}(k)-(\delta+n)+\tau k f^{\prime \prime}(k)-\frac{\pi^{\prime}(\tau \omega(k)) \tau \omega^{\prime}(k)\left[(1-\tau) f(k)+\tau k f^{\prime}(k)\right]}{1+n+\pi(\tau \omega(k))}\right] \\
& \lim _{k \rightarrow \infty} \phi^{\prime}(k)=\lim _{k \rightarrow \infty}\left[f^{\prime}(k)-(\delta+n)+\tau k f^{\prime \prime}(k)\left(1+\frac{\pi^{\prime}(\tau \omega(k))\left[(1-\tau) f(k)+\tau k f^{\prime}(k)\right]}{1+n+\pi(\tau \omega(k))}\right)\right]
\end{aligned}
$$

Given that $f^{\prime \prime}(k)<0$, the condition $\lim _{k \rightarrow \infty} \phi^{\prime}(k)<0$ is always true when $\lim _{k \rightarrow \infty} f^{\prime}(k)-$ $(\delta+n)<0$.

Hence, under the two conditions of the Proposition, the function $\phi(k)$ reaches a maximum between 0 and $+\infty$. Given that the function $\phi(k)$ is continuous, this maximum $k_{\mathrm{GR}}$ satisfies $\phi^{\prime}\left(k_{\mathrm{GR}}\right)=0$.

To better understand the Proposition it is useful to compare with the case in which longevity is exogenous, i.e. $\pi^{\prime}(h)=0$ and $\tau=0$. The condition of the proposition would collapse to

$$
\lim _{k \rightarrow+\infty} f^{\prime}(k)<\delta+n<\lim _{k \rightarrow 0+} f^{\prime}(k)
$$

which is assumption A5 in de la Croix and Michel (2002). However, under endogenous longevity, the interiority of the Golden Rule capital level requires a stronger condition regarding the level of $\lim _{k \rightarrow 0+} f^{\prime}(k)$. The intuition behind the additional term in the condition is that the interiority of the Golden Rule requires also, in the context of endogenous longevity, that a small increase of capital in the neighbourhood of 0 does not lead to an explosion of the population through a rise of the survival probability, in which case the optimal capital level would be zero.

Under exogenous longevity $\pi()=.\bar{\pi}$, equation (7) would simplify into:

$$
\phi^{\prime}(k)=\frac{\left[f^{\prime}(k)-(\delta+n)\right](1+n)}{1+n+\bar{\pi}}
$$

and the Golden Rule $\bar{k}_{\mathrm{GR}}$ satisfies

$$
f^{\prime}\left(\bar{k}_{\mathrm{GR}}\right)=\delta+n
$$

The Golden rule capital level with exogenous longevity is independent from the postulated level of the probability of survival $\pi$. However, it is important to stress that 
the level of consumption per head for a given capital level is not independent from the level of $\pi$. Although it is for the same level of capital per worker that steady-state consumption per head is maximized, the level of the consumption profile is higher the lower $\pi$ is. The intuition behind this is that $\pi$, by increasing the population size, reduces consumption per head per period of life for a given level of $k$. Thus, although $\pi$ does not affect the Golden Rule capital level, it does influence the level of consumption per head under each capital level.

Proposition 2 The Golden Rule capital level under endogenous longevity $k_{\mathrm{GR}}$ is inferior to its level under exogenous longevity $\bar{k}_{\mathrm{GR}}$.

Proof. Let us consider condition $\phi^{\prime}\left(k_{\mathrm{GR}}\right)=0$ in (8). Under exogenous longevity, $\pi^{\prime}(h)=$ 0 , so that the RHS equals 0 , and health expenditures equal also 0 (i.e. $\tau=0$ ), so that we are back to the conventional Golden Rule, stating that consumption per head is maximized when the marginal productivity of capital equals $\delta+n$, so that the numerator of the LHS equals 0, and the LHS equals the RHS.

However, under endogenous longevity, the RHS differs from 0, and is strictly positive. Hence, it must also be the case that $f^{\prime}\left(k_{\mathrm{GR}}\right)-(\delta+n)+\tau\left[k_{\mathrm{GR}} f^{\prime \prime}\left(k_{\mathrm{GR}}\right)\right]$ is positive, so that:

$$
f^{\prime}\left(k_{\mathrm{GR}}\right)-(\delta+n)>\tau\left[-k_{\mathrm{GR}} f^{\prime \prime}\left(k_{\mathrm{GR}}\right)\right]
$$

Given that the RHS is strictly positive, this inequality implies that the marginal productivity of capital must here be strictly larger than its level under exogenous longevity. Thus, it follows that the Golden Rule capital level must be smaller than under exogenous longevity.

The intuition behind that result goes as follows. In a Chakraborty-type economy, raising capital per worker tends also to increase, through $h(k)$, the proportion $\pi$ of survivors in the cohort, and, thus, the population size (unlike what happens in economies where longevity is exogenous). Hence, under that additional effect, the level of $k$ maximizing steady-state consumption per head must be inferior to its level under exogenous longevity.

Regarding the uniqueness of the Golden Rule capital level $k_{\mathrm{GR}}$, let us notice that it is not guaranteed in the present context, unlike what prevails under exogenous longevity (under exogenous longevity, we have $\phi^{\prime \prime}(k)=f^{\prime \prime}(k)$, which is negative, so that $\phi(k)$ is concave for all values of $k$ ). To see this, it is sufficient to realize that the second order derivatives $\phi^{\prime \prime}(k)$ depends on the second order derivatives of the survival function $\pi$ and on the third-order derivative of the production function via $\omega^{\prime \prime}$, for which we have no reasonable assumption to impose. It is therefore not necessarily negative, contrary to what prevails when longevity is exogenous.

An example illustrates this point. Let us take a Cobb-Douglas production function $f(k)=10 k^{1 / 3}$. Assume a tax rate $\tau=0.15$, full depreciation of capital $\delta=1$ and constant population $n=0$. The Golden Rule is $\bar{k}_{\mathrm{GR}}=6.086$. Consider two different cases for the survival function $\pi(h)$. One concave, $\pi(h)=h /(1+h)$, as in Chakraborty 
Figure 1: Net production $\phi(k)$ with a concave survival function $\pi(h)$ (left panel) and with a logistic one (right panel)
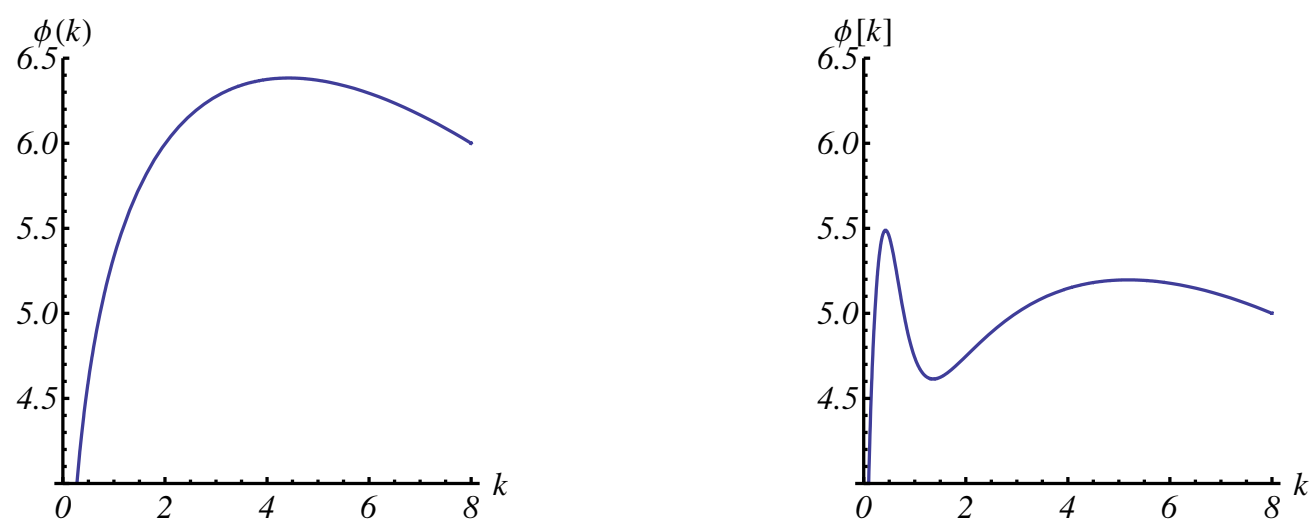

(2004), and one logistic $\pi(h)=0.0001 /(0.0001-\exp (-10 h))$. The left panel of Figure 1 shows that net production is globally concave in the first example and the first order condition $\phi^{\prime}\left(k_{\mathrm{GR}}\right)=0$ gives a global maximum with $k_{\mathrm{GR}}=4.42$. In the right panel, net production is concave-convex-concave. We have two local maxima, 0.428 and 5.182, and the one with the smallest $k$ is the global maximum.

\section{The Lifetime Golden Rule}

When interpreting the above results, one may argue that the maximization of consumption per head per period of life is not an adequate goal, in the sense that it only captures the intensity of life's goodness (i.e. in per period terms), but not the goodness of life as a whole.

On the contrary, under the assumption of endogenous lifetime, a more adequate goal may be the maximization of expected lifetime consumption per head. Let us now derive the capital level maximizing expected lifetime consumption per head, defined as the consumption per head multiplied by life expectancy:

$$
\frac{C_{t}}{L_{t}+\pi_{t} L_{t-1}}\left(1+\pi_{t}\right)=c_{t} \frac{(1+n)\left(1+\pi_{t}\right)}{1+n+\pi_{t}}
$$

Expected lifetime consumption per head at the steady-state, denoted by $\psi(k)$, is:

$$
\psi(k) \equiv c \frac{(1+n)(1+\pi)}{1+n+\pi}=[f(k)-k(\delta+n)-\tau \omega(k)] \frac{(1+n)(1+\pi)}{1+n+\pi}
$$

The existence of a (finite positive) level of $k$ maximizing $\psi(k)$ would be guaranteed under the conditions insuring that $\psi^{\prime}(k)$ is positive for low capital levels but negative for high ones. Those conditions coincide with the ones implying $\phi^{\prime}(k)>0$ for $k$ tending towards 0 and $\phi^{\prime}(k)<0$ for $k$ tending towards $+\infty$. 
Proposition 3 Under the conditions of Proposition 1, there exists a capital per worker maximizing the expected lifetime consumption per head at the steady-state. That Lifetime Golden Rule capital level, denoted by $k_{\mathrm{LGR}}$, is such that:

$$
\frac{f^{\prime}\left(k_{\mathrm{LGR}}\right)-(\delta+n)+\tau k_{\mathrm{LGR}} f^{\prime \prime}\left(k_{\mathrm{LGR}}\right)}{f\left(k_{\mathrm{LGR}}\right)-k_{\mathrm{LGR}}(\delta+n)-\tau \omega\left(k_{\mathrm{LGR}}\right)}=\frac{n}{1+\pi\left(\tau \omega\left(k_{\mathrm{LGR}}\right)\right)} \frac{\pi^{\prime}\left(\tau \omega\left(k_{\mathrm{LGR}}\right)\right) \tau k_{\mathrm{LGR}} f^{\prime \prime}\left(k_{\mathrm{LGR}}\right)}{1+n+\pi\left(\tau \omega\left(k_{\mathrm{LGR}}\right)\right)} .
$$

We have that: if $n \leq 0, \bar{k}_{\mathrm{GR}}<k_{\mathrm{LGR}}$ and $k_{\mathrm{LGR}}<k_{\mathrm{GR}}$; if $n>0, \bar{k}_{\mathrm{GR}}<k_{\mathrm{LGR}}$ and $k_{\mathrm{LGR}}<k_{\mathrm{GR}}$ or $k_{\mathrm{LGR}}>k_{\mathrm{GR}}$

Proof. Different cases should be distinguished here, depending on the sign of $n$. Under $n=0$, the LHS is the same as above, but the RHS equals 0, so that the Lifetime Golden Rule capital level must exceed the Golden Rule capital level. However, given that we have

$$
f^{\prime}\left(k_{\mathrm{LGR}}\right)-(\delta+n)=\tau\left[-k_{\mathrm{LGR}} f^{\prime \prime}\left(k_{\mathrm{LGR}}\right)\right]>0
$$

it follows that the Lifetime Golden Rule capital is here lower than under exogenous longevity.

Under $n>0$, the RHS is now negative, so that:

$$
f^{\prime}\left(k_{\mathrm{LGR}}\right)-(\delta+n)<\tau\left[-k_{\mathrm{LGR}} f^{\prime \prime}\left(k_{\mathrm{LGR}}\right)\right]>0
$$

from which one can see that $k_{\mathrm{LGR}}$ must necessarily exceed the standard Golden Rule capital level. However, $k_{\mathrm{LGR}}$ may or may not exceed the Golden Rule capital under exogenous longevity $k_{\mathrm{GR}}$, defined in such a way that $f^{\prime}\left(k_{\mathrm{GR}}\right)-(\delta+n)=0$, because the LHS of the above expression can be positive or negative. A similar reasoning could be applied to the case $-1<n<0$.

Note that, here again, the first-order condition would, under an exogenous longevity i.e. $\pi^{\prime}(h)=0$ and $\tau=0$ - vanish to the standard Golden Rule $f^{\prime}\left(k_{\mathrm{LGR}}\right)=\delta+n$. Back to the general case where longevity is endogenous, it should be stressed that, in comparison with the condition characterizing the Golden Rule capital level, the LHS is exactly the same, whereas the RHS is now multiplied by $-n /(1+\pi)$.

\section{Concluding remarks}

Endogenizing longevity does not leave the Golden Rule of capital accumulation unchanged. Clearly, if the goal is the maximization of steady-state consumption per head, the Golden Rule capital level is inferior to its level under exogenous longevity, as raising $k$ increases the population size through a higher survival to the second period. Hence, the endogeneity of longevity tends here to qualify the extent of underaccumulation of capital, as the 'target' of capital becomes lower.

Given that one may not be fully satisfied with the goal of maximization of consumption per head (as this leaves longevity aside), we also characterize the Lifetime Golden Rule 
capital level, which maximizes the expected lifetime consumption per head. The Lifetime Golden Rule capital level is superior to the standard Golden Rule capital level under endogenous longevity. Hence, shifting to the goal of expected lifetime consumption maximization reinforces - rather than weakens - the likelihood of underaccumulation of capital in comparison with the goal of maximization of consumption per period. But the Lifetime Golden Rule capital level may be inferior or superior to the Golden Rule capital under exogenous longevity.

Finally, it should be stressed that this study does not rely on assumptions on preferences. Ideally, the best social objective consists of the Golden Age, i.e. the capital per worker maximizing steady-state lifetime utility. However, its definition is not trivial, as this requires to deal with some necessary assumptions on the utility of death, unlike what was needed in the study of the Golden Rule and Lifetime Golden Rule capital levels. The examination of the Golden Age under various assumptions on the utility of death is on our research agenda.

\section{References}

Boucekkine, R. (2008): "Growth Economics of Epidemics: A Review of the Theory" Mathematical Population Studies, 15, pp. 1-26.

Cervellati, M. and Sunde, U. (2005): "Human capital formation, life expectancy, and the process of development", American Economic Review, 95, pp. 1653-1672.

Chakraborty, S. (2004): "Endogenous lifetime and economic growth", Journal of Economic Theory, 116, pp. 119-137.

Chakraborty, S. and Das, M. (2005): "Mortality, human capital and persistent inequality", Journal of Economic Growth, 10, pp. 159-192.

de la Croix, D. \& Michel, P. (2002): A Theory of Economic Growth. Dynamics and Policy in Overlapping Generations. Cambridge University Press, Cambridge.

Diamond, P. (1965): "National debt in a neoclassical growth model", American Economic Review, 55(5), pp. 1126-1150.

Phelps, E. (1961): "The golden rule of accumulation: a fable for growthmen", American Economic Review, 51(4), pp. 638-643.

Phelps, E. (1965): "Second essay on the golden rule of accumulation", American Economic Review, 55(4), pp. 793-814. 
Département des Sciences Économiques de l'Université catholique de Louvain

Institut de Recherches Économiques et Sociales

Place Montesquieu, 3

1348 Louvain-la-Neuve, Belgique 